\title{
THE MARCH MEETING IN CHICAGO
}

The two hundred sixty-ninth regular meeting of the Society was held at the University of Chicago on Friday and Saturday, March 29-30, 1929. About one hundred fifty persons attended the meeting, among whom were the following one hundred fourteen members of the Society, the largest number of members ever attending a meeting in Chicago:

R. W. Babcock, Bardell, Barnard, Barnett, Bartky, Beenken, Bibb, G. A. Bliss, Boyce, Brand, Brixey, O. E. Brown, L. H. Bunyan, Butler, Cairns, G. N. Carmichael, R. D. Carmichael, E. H. Carus, Chittenden, Coble, H. H. Conwell, Copp, H. V. Craig, Curtiss, Dancer, H. A. Davis, Dickson, P. D. Edwards, Eiesland, Escott, H. P. Evans, Everett, Feltges, Georges, Germond, Gilmore, J. W. Glover, Gouwens, L. M. Graves, Griffiths, V. G. Grove, Hartung, Hawkins, E. R. Hedrick, Holgate, Louis Ingold, Ingraham, Dunham Jackson, Jonah, B. W. Jones, Kern, Ketchum, Knight, E. P. Lane, Langer, LaPaz, Larsen, Latimer, Longley, McCoy, McNair, MacDuffee, MacMillan, March, T. E. Mason, Merriman, E. H. Moore, Max Morris, D. C. Morrow, E. J. Moulton, Musselman, Oakley, Olson, Palmer, Parkinson, Pepper, Powell, Price, Rainich, Rashevsky, R. G. D. Richardson, H. L. Rietz, Ross, Roth, Rowland, Sanger, Schottenfels, Sheets, Shohat, R. C. Shook, W. G. Simon, Skinner, Slaught, E. R. Smith, Sokolnikoff, Speeker, Stafford, Stouffer, M. E. Taylor, Teach, J. S. Turner, Van Vleck, Vass, Wahlin, Wall, L. E. Ward, Warren Weaver, Weiss, Wiggin, R. L. Wilder, Wiley, K. P. Williams, F. E. Wood, F. L. Wren.

On Friday afternoon Professor E. B. Van Vleck gave a symposium address on The location of roots of polynomials and entire functions. A rising vote of thanks was given the speaker.

On Friday evening members and their guests attended a dinner in the Del Prado Hotel. Professor Holgate was toastmaster. He called upon Professor R. G. D. Richardson who spoke of the aspirations and future plans of the Society. Professor G. A. Bliss then spoke in appreciation of the scientific work and influence of Professor Van Vleck, and the toastmaster read a letter from Professor Birkhoff on the same subject. Professor Holgate then called on Professor Van 
Vleck, and afterwards on Professor E. H. Moore. The last speaker was President Hedrick, who spoke of Professor Van Vleck's work and its lessons for the future. The dinner was attended by ninety-four persons. There was a telegraphic exchange of greetings between the groups meeting in Chicago and in New York.

Sessions of the Council were held both before and after the dinner, the President and Secretary being in attendance.

It was announced that the following twenty-eight persons were elected to membership:

Professor Edwin Brown Allen, Rensselaer Polytechnic Institute;

Miss Jean C. Hempstead, Drexel Institute;

Mr. Everett H. Johnson, Lehigh University;

Mr. Paul Allen Knedler, University of Pennsylvania;

Mr. Clarence Anding Lovell, Drexel Institute;

Mrs. Elizabeth Elliott Nixon (Mrs. J. C.), University of Alabama;

Miss Irene Price, Indiana University;

Mr. John Albert Roulton, engineer, Rainey-Wood Coke Company, Swedeland, Pa.;

Mr. Ronald G. Smith, Kansas University;

Professor Guy Stevenson, University of Louisville;

Mr. Alexander Tartler, Drexel Institute;

Mr. Charles Henry Vehse, Brown University;

Mr. Ralph Oaks Virts, Indiana University;

Professor Horatio Burt Williams, Columbia University;

Nominees of the Mathematical Association of America:

Professor I. F. Neff, Drake University, Des Moines, Iowa;

Mr. J. C. Nixon, University of Alabama, Tuscaloosa, Ala.;

Mr. R. E. Peterson, Pennsylvania State College, State College, Pa.;

Nominees of the University of Pennsylvania:

Mr. V. W. Adkisson; Mr. Leonard Carlitz; Mr. Alexander G. Makarov;

Nominees of the Metropolitan Life Insurance Company:

Mr. George V. Brady; Mr. J. Christman; Mr. Reinhard A. Hohaus;

Nominees of Members of the Department of Mathematics, University of Wisconsin:

Mr. R. Erickson; Mr. H. H. Germond; Mr. E. Hawkins; Mr. H. D. Larsen; Mr. J. S. McNair.

In pursuance of the action taken at the Annual Meeting of 1928, the San Francisco Section has been abandoned. Professor B. A. Bernstein was appointed as Associate Secre- 
tary and Professors M. W. Haskell (chairman), E. T. Bell, H. F. Blichfeldt, and R. M. Winger on the Committee on Program to work with him, in organizing the future meetings of the Society on the Pacific slope.

It was decided that the meetings of the Southwestern Section be stopped and that the following working plan govern the allocation of meetings in the Middle West:

1. In case the Western Meeting of the Society in connection with the Association for the Advancement of Science in December is held in one of the two regions southeast of Chicago or southwest of Chicago, a meeting of the Society on the Saturday after Thanksgiving of the same year shall be held in the other region.

2. In case the above mentioned December meeting of the Society is not held in either region during a given year, but the Summer Meeting of the Society that year is held in one of the two regions, a meeting of the Society shall be held that year on the Saturday after Thanksgiving in the other region.

3. In case neither the December Meeting nor the Summer Meeting is held in one of the two regions during a given year, the Council shall authorize a meeting to be held on the Saturday after Thanksgiving that year in either the region southeast or the region southwest of Chicago, somewhat in rotation.

There are now no sections remaining and all meetings held will be meetings of the Society; hereafter all meetings are to be numbered seriatim and subsidiary numbering abandoned.

The 1929 Thanksgiving meeting of the Western group is to be held at Ann Arbor. Invitations from President Vinson of Western Reserve University and President Howe of Case School of Applied Science to hold a meeting in Cleveland in connection with the 1930-31 meeting of the American Association for the Advancement of Science were accepted.

The following appointments were made: as a committee to nominate officers and members of the Council: Professors O. D. Kellogg (chairman), C. R. Adams, R. D. Carmichael, L. E. Dickson, and Tomlinson Fort; as a committee for the 
meeting in Ann Arbor: Professors J. W. Glover (chairman), J. W. Bradshaw, T. H. Hildebrandt, M. H. Ingraham, and W. G. Simon; as a committee for the meeting in Cleveland: Dean T. M. Focke (chairman), Professors W. D. Cairns, W. B. Ford, M. H. Ingraham, and W. G. Simon; to represent the Society on the Council of the American Association for the Advancement of Science for 1929: Professors Louis Ingold and M. H. Ingraham; to represent the Society on the American Year Book: Professor Tomlinson Fort.

Mr. Charles A. Cole has made a gift to the Society, in memory of his father, which more than doubles the Frank Nelson Cole Prize Fund. In thanking him, the Council expressed its conviction that this generous action will prove an important factor in the furtherance of those high ideals of the Society toward the establishing of which the father so notably contributed.

A memorandum of the financial needs of the Society as prepared at the request of the General Education Board was presented to the Council.

Minor changes in the by-laws were recommended to the Society for action at the Summer Meeting.

In view of the tremendous demands for space in the Bulletin, the editors of the Bulletin and Transactions were requested to study the problem of journal publication for the Society. The appointment also was authorized of a committee to investigate the demand and the probable financial support for a journal of applied mathematics.

The papers whose abstracts appear below were read in three sections: (1) Analysis-Friday morning; (2) Geometry and Point Sets-Friday morning; (3) General-Saturday morning. President Hedrick, Professors Van Vleck and Bliss presided at the first; Professors Lane and Ingold at the second; President Hedrick and Professor Rietz at the third. Papers 1-14 were read in the first section; papers $15-23$ in the second; and papers $24-39$ in the third. Papers 10, 11, 13, $14,23,35-38$ were read by title. 
1. Dr. H. S. Wall: On the Pade table of approximants associated with a positive definite power series.

This paper studies the diagonal files in the Padé table for series in which the Stieltjes inequalities $A_{n}>0, B_{n}>0$, are replaced by the single condition $A_{n}>0$. The results are somewhat analogous to those obtained by the writer for the less general Stieltjes series (Transactions of this Society, vol. 31 (1929), pp. 91-116). Let $S_{0}, S_{1}, S_{2}, \cdots$ denote the principal diagonal file and the successive parallel files to the right of it respectively, in the Padé table. Then all the files (1) $S_{0}, S_{2}, S_{4}, \cdots$ converge to limits which are analytic over any region not containing a part of the real axis. The files $S_{1}, S_{3}, \cdots$ need not converge. There are just three cases. Case i, all the files (1) have a common limit; Case ii, the files $S_{0}, S_{2}, \cdots, S_{2 k}$ have a common limit while no two of the subsequent files have equal limits; Case iii, no two of the files (1) have equal limits. Similar results are obtained for the files below the principal diagonal. In cases ii, iii the limits are all meromorphic functions of $1 / z$.

2. Dr. H. S. Wall: On the expansion of an integral of Stieltjes.

This paper studies the formal expansion in descending powers of $z$ of the integral $\int_{0}^{\infty}\left[(1-\lambda) /\left(e^{(1-\lambda) u}-\lambda\right)\right] e^{-z u} d u$, a continued fraction development of which was given by Stieltjes, Recherches sur les fractions continues (Oeuvres, vol. 2 , p. 547$)$. The $(n+1)$ st coefficient in this expansion is a reciprocal polynomial of degree $n-1$ in $\lambda: c_{n}(\lambda)=\sum_{i=0}^{n-1} a_{n}, i_{i}{ }^{i}$, having only real, negative, simple roots, the roots of $c_{n}$ being separated by those of $c_{n-1}$. Since $c_{n}(1)=n$ !, the coefficients constitute a partition of $n$ !, the number of elements in the symmetric group $G_{n !}$. Let $t$ take $k$ into $i_{k}$. Let $K_{n, p}$ denote the set of substitutions $t$ in which exactly $p$ inequalities $i_{k+1}>i_{k}$ obtain. Then $K_{n, p}$ contains $a_{n, p}$ elements and $T^{-1} K_{n, p} T=K_{n, p}$, where $T=(1, n)(2, n-1)(3, n-2) \cdots$. The sets $K_{n, p}$ and $K_{n, n-p-1}$ contain the same number of conjugate pairs $e_{p}, \bar{e}_{p}=T^{-1} e_{p} T$ and $e_{n-p-1}$, $\bar{e}_{n-p-1}=T^{-1} e_{n-p-1} T$ respectively, and also equal numbers of elements $g_{p}, g_{n-p-1}$, respectively, self-conjugate with respect to $T$. The set of all elements $g$ forms a group of order $k ! 2^{k}$ when $n=2 k$ or $2 k+1$. Certain multiplication laws hold among the four elements $e_{p}, T e_{p}=e_{n-p-1}, \bar{e}_{p}$, and $T \bar{e}_{p}=\bar{e}_{n-p-1}$ and also among the elements $g_{p}, T g_{p}=g_{n-p-1}$ and $g_{p}^{\prime}, T g_{p}^{\prime}=g_{n-p-1}^{\prime}$.

3. Professor J. A. Shohat: On a certain formula of mechanical quadratures with non-equidistant ordinates.

The author investigates the following formula of mechanical quadratures:

$$
\int_{-n}^{n} u_{x} d x=a\left(u_{-n}+u_{n}\right)+\sum_{i=1}^{n} a_{i}\left(u_{-n_{i}}+u_{n_{i}}\right), \quad\left(u_{x} \equiv u(x)\right) ;
$$

$\left(0<n_{i}<n\right)$, which has been discussed for $s=2,3$ by G. F. Hardy, A. King and J. Glover. The existence of (A) is established for any positive integer $s$, 
also its convergence for $s \rightarrow \infty$; the properties of the $n_{i}(s)$ and $a_{i}(s, a)$, also their numerical values for $s=3,4$ are given. This paper will appear in full in the Transactions of this Society.

\section{Professor G. A. Bliss: An integral inequality.}

This paper contains a proof for $n>m>1$ of the inequality

$$
\int_{0}^{\infty} y^{n} d x / x^{n-r} \leqq\left(\int_{0}^{\infty} f^{m} d x\right)^{n / m} \quad(r=n / m-1),
$$

when the integral in the second member exists in the sense of Lebesgue and

$$
y=\int_{0}^{x} f d x, \quad k=(1 /(n-r-1))[r \Gamma(n / r) / \Gamma(1 / r) \Gamma(n-1 / r)] r .
$$

The functions $f$ for which the equality holds are specified. The method used constitutes a very interesting application of the calculus of variations to a case which is somewhat irregular. The inequality was suggested by Professor G. H. Hardy, and the paper is in answer to a query from him as to whether a proof by calculus of variations methods is possible. No other proof has as yet been given so far as is known to the writer.

\section{Dr. Lincoln La Paz: The Hamiltonian function and an inverse problem of the calculus of variations.}

The problem solved is the following: Given a function $H\left(x, y_{1}, \cdots\right.$, $\left.y_{n}, u_{1}, \cdots, u_{n}\right)$ arbitrary except for suitable continuity properties, under what conditions is this function the Hamiltonian function of a problem of the calculus of variations in $\left(x, y_{1}, \cdots, y_{n}\right)$-space? Necessary and sufficient conditions are found to be that $H$ be of class $C^{\prime \prime \prime}$ in a region $S$ of $\left(x, y_{1}, \cdots, y_{n}, u_{1}, \cdots, u_{n}\right)$ values and that the determinant $\left|\partial^{2} H / \partial u_{k} \partial u_{m}\right|$ be different from zero in $S$. The method used leads to an explicit expression for the integrand function of the variation problem associated with an admissible function $H$. As a corollary we obtain the conditions under which a prescribed first-order partial differential equation of the form $F\left(x, y_{1}, \cdots, y_{n}, W_{x}, W_{y_{1}}, \cdots, W_{y_{n}}\right)=0$ can be the Hamilton-Jacobi partial differential equation of a problem of the calculus of variations in $\left(x, y_{1}, \cdots, y_{n}\right)$-space. Paulus has devoted a paper (Mathematische Zeitschrift, vol. 25 (1926), pp. 348-361) to the solution of this last problem in the special case $n=1$. His argument is based on properties of the Hilbert invariant integral rather than of the Hamiltonian function.

6. Dr. P. W. Ketchum: Solution of partial differential equations by hypervariables.

Consider the differential operator $P\left(p_{1}, \cdots, p_{n}\right)$, where $p_{i}=\partial / \partial w_{i}$, and $P$ is a homogeneous polynomial of degree $r$, with constant coefficients. The necessary and sufficient conditions that the real part of all analytic functions $F(w)$, of the hypervariable $w=\sum w_{i} e_{i}$, be a general solution of the equation $P \cdot f\left(w_{1}, \cdots, w_{n}\right)=0$ are that $P\left(e_{1}, \cdots, e_{n}\right)=0$, and that the real part of $\alpha w^{t}$ contain a sufficient number of arbitrary constants. The number of such constants depends on $n$ and on $r$, and, if $n$ is greater than 2, the num- 
ber increases indefinitely with $t$. It is thus seen why it is necessary to use an infinite hypervariable for Laplace's equation. By application of the above theorem, hypervariables were found which furnish general solutions of the wave equation and of Airy's equation.

\section{Professor L. E. Ward: On the region of convergence of a} certain type of series.

The series considered is that consisting of the characteristic functions of the irregular boundary value problem $d^{3} u / d x^{3}+\rho^{3} u=0, u(\pi)=u\left(-\omega_{2} \pi\right)$ $=u\left(-\omega_{3} \pi\right)$. The author has shown (Transactions of this Society, Oct., 1927 , pp. 741-745) that if such a series converges uniformly in any segment of the interval $(0, \pi)$, it will converge uniformly in and on any equilateral triangle $T$ centered at $x=0$ and having one vertex at an interior point of the segment of assumed uniform convergence; and also that any function analytic in a region containing $x=0$ as an interior point can be expanded in such a series uniformly convergent in and on a certain triangle $T$. By examining the magnitude of the coefficients of the formal expansion of a function, the author shows in the present paper that if the function is analytic in a region of which $x=0$ is an interior point and also satisfies certain continuity conditions, the triangle of uniform convergence can be taken as large as one likes except that it shall not contain within or upon itself either a singularity of the function or the point $\pi$.

\section{Professor P. D. Edwards: Functions possessing an ad-} dition theorem from the standpoint of a functional equation given by Abel.

The usual procedure in the study of functions having addition theorems has been to define a function $\theta(x)$ and then to determine whether or not there exists a function $f$ such that $\theta(x+y)=f(\theta(x), \theta(y))$. This paper inverts the problem and looks at it from the standpoint of the function $f$. A necessary and sufficient condition that $f$ represent an addition theorem is that it satisfy a functional equation given by Abel. This functional equation is used to find explicit formulas for $f$ in the case where $f$ is rational and in certain irrational cases. The function $\theta$ corresponding to a given $f$ is found by the aid of a theorem due to Weierstrass, and thus the addition theorems of most of the well known functions are obtained.

9. Professor Louis Brand: On the transformation of surface integrals to circuit integrals.

The parent theorem of all transformation of this class is

$$
\int n \times F d \sigma=\int t F d s,
$$

where $F$ is a scalar, vector or tensor function of position and $\nabla F$ is either a surface or space gradient. When $F$ is a tensor function, many other theorems follow from this by equating invariants of both members which are distributive with respect to addition. One such theorem is 


$$
\int\left\{\nabla F \cdot n^{(2)}-n \operatorname{div} F\right\} d \sigma=\int t \times F d s,
$$

where the index 2 means that the operation $\cdot n$ applies to the second vector of the tensor preceding and div $F$ denotes a surface divergence if $\nabla F$ is a surface gradient. Now if $f$ is a second function of the same generality as $F$ and we put $F=n f$ in (2), we obtain the integral transformation

$$
\int(\nabla f+J n f) d \sigma=\int t \times n f d s
$$

$J$ being the mean curvature. This transformation is coextensive with (1); this may be shown by putting $f=n F$ in (3) and equating cross invariants. From (3) in turn other useful theorems may be deduced by the process of equating distributive invariants. One of these theorems finds an important application in the problem of minimizing a surface integral limited by a fixed closed curve.

10. Mr. W. T. Reid: A boundary-value problem for a countable system of ordinary linear differential equations.

The system considered is $(1) y^{\prime}(x, \mu)=A(x, \mu) y(x, \mu)$ where $y(x, \mu)$ is an absolutely continuous vector in Hilbert space on $X: 0 \leqq x \leqq 1$ and for values of the parameter $\mu$ on $L: L_{1}<\mu<L_{2}$ the matrix $A$ is summable on $X$ and limited by the summable function $\phi(x)$ uniformly for $\mu$ on $L$; furthermore, for $x$ almost everywhere on $X$ and $\mu$ on $L, A(x ; \mu)$ is a vollstetig matrix, as defined by Hilbert. This system with the following boundary conditions (2) $[E+G(\mu)] y(0 ; \mu)+[E+H(\mu)] y(1 ; \mu)=0$, where $E$ is the unit matrix, $G$ and $H$ are "vollstetig" matrices and $E+G$ and $E+H$ have unique reciprocals for each value of $\mu$, has many of the properties of a finite system. When it is incompatible we may define uniquely a Green's matrix. It is proved that if for $\mu=\mu^{\prime}$, system (1), (2) is compatible there exists a reduced system $\left(1^{\prime}\right),(2)$ which is incompatible, where $\left(1^{\prime}\right), y^{\prime}(x)=\left[A\left(x ; \mu^{\prime}\right)+P(x)\right] y(x)$ and $P$ is a matrix of non-negative continuous functions which is limited on $X$ by an arbitrarily assigned $\epsilon$.

\section{Mr. W. T. Reid: Boundary conditions for a countable system of linear differential equations.}

Two infinite matrices $M$ and $N$ that are limited, as defined by Hilbert, are said to belong to class $D_{1}\left[D_{2}\right]$ if there exists an infinite matrix $\left(M^{\prime}, N^{\prime}\right)$ [ $\left.\left(\begin{array}{c}M^{\prime} \\ N^{\prime}\end{array}\right)\right]$ composed of columns [rows] of $M$ and $N$ which possesses a unique reciprocal, and furthermore there exist an infinite number of columns [rows] of $M$ and $N$ which do not belong to $\left(M^{\prime}, N^{\prime}\right)\left[\left(\begin{array}{l}M^{\prime} \\ N^{\prime}\end{array}\right)\right]$. We consider the infinite system $y^{\prime}(x)=A(x) y(x)$, where $y(x)$ is an absolutely continuous vector in Hilbert space and the matrix $A(x)$ is summable and limited by a summable function $\phi(x)$ on $X: 0 \leqq x \leqq 1$, with boundary conditions $M y(0)+N y(1)=0$, where the matrices $M$ and $N$ are of class $D_{1}$. It is shown that for the adjoint system $z^{\prime}(x)=-z(x) A(x)$ it is possible to determine boundary conditions $z(0) P+z(1) Q=0$, where $P$ and $Q$ are matrices of class $D_{2}$ and $M P-N Q=0$. 
Such a definition of adjoint boundary conditions reduces for a finite system to that given by Bounitzky.

12. Professor H. W. March: The field of a magnetic dipole in the presence of a conducting sphere.

A plane coil through which an alternating current is flowing may be regarded as an alternating magnetic dipole at distances from the coil which are great in comparison with its dimensions. In this paper the general problem of the field of such a dipole in the presence of a conducting sphere is solved. From the general formulas, approximate expressions are obtained for the field components for the case in which the radius of the sphere is small in comparison with the wave length.

\section{Dr. J. M. Earl: The degree of convergence of Hermite} and Laguerre series.

In a previous paper dealing primarily with a more general problem (Bulletin of this Society, vol. 34, p. 416), the writer has discussed incidentally the degree of convergence of the Hermite development of a given function, the treatment being based directly on the fact that the partial sum $S_{n}(x)$ of order $n$ is a polynomial of the $n$th degree which makes the integral of $e^{-x^{2}}\left[f(x)-S_{n}(x)\right]^{2}$ from $-\infty$ to $+\infty$ a minimum. By use of an asymptotic expression for the Hermite polynomial of degree $n$, it is now shown that on any finite interval, $S_{n}(x)$ does not exceed a constant multiple of $M \log n$, where $M$ is the least upper bound of $|f(x)| e^{-h x^{2}}$ for all real values of $x$, and $h$ is any given number less than $1 / 2$. This leads to improved results with regard to the degree of convergence of the Hermite series. Corresponding treatment is applicable to the Laguerre development on the simply infinite interval.

14. Mr. E. L. Mickelson: On the approximate representation of a function of two variables.

The first four sections of this paper extend known theorems on finite trigonometric and polynomial sums approximating a function of one variable which possesses a modulus of continuity to the case of a function of two variables which is similarly restricted. The first two consist of theorems on the existence of trigonometric and polynomial approximating double sums, the third treats double sums which are trigonometric in one variable and polynomial in the other, and the fourth applies the foregoing to discussion of the degree of convergence of certain double series-Fourier, Legendre, and a combination of these. In general terms, the fifth section proves that if a function possesses $r$ th order directional derivatives of a certain type which have a modulus of continuity, $\omega(d)$, on the surface of a unit sphere, the sum of the first $m+1$ terms of Laplace's series for the function approximates it to the order of $\omega(2 \pi / m) m^{1 / 2}(1 / m)^{r}$, for all finite, positive, integral values of $r$, including $r=0$. 


\section{Professor J. R. Musselman: A classification of planar} six points.

The author has made a classification of six points in the plane according to equalities existing between their irrational invariants. These invariants were developed by Coble, Transactions of this Society, vol. 16 (1915). In the present paper the author has divided six points into a certain number of types and developed canonical forms for them.

16. Dr. H. A. Davis: Non-involutorial transformations belonging to a non-special linear complex.

A brief synthetic discussion of non-involutorial transformations belonging to a non-special linear complex has been given by D. Montesano. The present paper extends the synthetic discussion and investigates the problem analytically. The equations of the general transformation are obtained and discussed.

17. Dr. H. A. Davis: Non-involutorial transformations belonging to a special linear complex.

The equations of the general non-involutorial transformation which belong to a special linear complex are here obtained and discussed. The analytic work reveals an error in the synthetic discussion of the problem by M. Pieri (Circolo Matematico di Palermo, vol. 6). This error causes a considerable part of Pieri's paper to be incorrect, especially the $F$ - and $P$ systems of the transformation. In the present paper the equations of the components of the latter are obtained and discussed. It is also shown that the general transformation can be built up from the $T_{3-3}$ in a plane through the directrix of the special linear complex. The study of this plane $T_{3-3}$ reveals some interesting facts concerning the $F$ - and $P$-systems of the space transformation.

\section{Professor E. P. Lane: On the contact of a quartic surface} with an analytic surface.

Since there are $(k+1)(k+2) / 2$ conditions for an algebraic surface to have contact of order $k$ at a point of an analytic surface, and since an algebraic surface of order $h$ depends on $h\left(h^{2}+6 h+11\right) / 6$ parameters, there is a limitation on the order of contact possible for a given algebraic surface. A quartic surface depends on 34 parameters, so that there is a six-parameter family of quartics having contact of the sixth order at a point of an analytic surface. It is the primary purpose of this paper to initiate an investigation of the contact of a general quartic surface with an analytic surface. The cases in which the quartics have contact of the fourth, fifth, and sixth orders receive particular attention. In each case the possibilities as to composite quartic surfaces are considered, and emphasis is laid on the curve of intersection of a non-composite quartic and the tangent plane at the point of contact. 


\section{Professor May M. Beenken: Surfaces in five-dimen- sional space.}

The method of Wilczynski is extended here to a systematic study of the projective differential geometry of surfaces in five-dimensional space $S_{6}$. A general surface in $S_{5}$ is determined, except for a projective transformation by a completely integrable system of four linear homogeneous partial differential equations of the third order. A local pyramid of reference is set up, and equations of linear osculants and of other linear spaces related to the surface are obtained in the local coordinate system. Quasi-asymptotic and principal curves, invented by the Italians as analogs of the asymptotic and conjugate curves on surfaces in ordinary space, are treated by a new method. Special surfaces in $S_{5}$, which sustain a conjugate net or one family of asymptotic curves, are investigated by means of other completely integrable systems of differential equations.

\section{Professor John Eiesland: On a class of ruled $(n-1)$ -} spreads in $S_{n}$, the $V_{4}^{4}$ in $S_{5}$.

The locus of all the $\infty^{n-2}$ lines that are incident with $n$ given generic $(n-2)$-flats in $S_{n}$ is a ruled $(n-1)$-spread. This spread has been studied by Segre for the case $n=4$. In a recent article in the Bulletin of this Society, vol. 34 (1928), B. C. Wong has constructed the generalized spread $V_{n-1}^{n-1}$ synthetically, but owing to the limitation of this method in the case of $n$ dimensional geometry, only a few of the more obvious properties of the spread have been obtained. In the present paper a strictly analytic method has been adopted. The equation of the general $V_{n-1}^{n-1}$ in $S_{n}$ has been obtained and a more detailed discussion of the geometric properties of the $V_{4}^{4}$ in $S_{5}$ is given. The introduction of the Grassmannian coordinates of a 3-flat in $S_{5}$ is a special feature of this part of the work.

\section{Professor R. L. Wilder: On the imbedding of subsets of a metric space in Jordan continua.}

Let $R$ be a metric space, and $M$ a subset of $R$. Several conditions which are both necessary and sufficient in order that $M$ should be a subset of a Jordan continuum ("continuous curve") $J$ of $R$ are obtained. Two of these follow: (1) If $M$ is compact and closed, it is necessary and sufficient that $M$ should be arcwise connected through $R$ as well as arcwise connected im kleinen through $R$. In this case, $J$ can be so chosen that $J-M$ is the sum of a denumerable set of non-overlapping open $\operatorname{arcs}\left\{L_{i}\right\}$ such that $\lim _{i \rightarrow \infty} d\left(L_{i}\right)=0$, and such that if $M$ has positive (Menger-Urysohn) dimension, $J$ is of the same dimension. (2) If $M$ is compact, it is necessary and sufficient that there should exist in $R$ a denumerable set of points $\left\{P_{i}\right\}$ which is dense on $M$, and such that the set of points $\left\{P_{i}\right\}$ is arcwise connected through $R$ as well as uniformly arcwise connected im kleinen through $R$. In this case, if the dimension of $M$ is $m$, and the dimension of the outer shell of $M$ is $s$, then $J$ may be so chosen that its dimension is the greater 
(or common) value of $m$ and $s$, provided, of course, that either $m$ or $s$ is positive.

22. Professor R. L. Wilder: On the relation of certain sets to their complements in euclidean spaces of three and higher dimensions.

This paper is an attempt to establish some fundamental relations between certain basic sets and their complements in euclidean spaces of three and higher dimensions. In particular, a result of R. L. Moore (Proceedings of the National Academy, vol. 4 (1918), p. 369) to the effect that each of the domains complementary to a simple closed curve in the plane is uniformly connected im kleinen is extended to higher spaces as follows: In $E_{n}$ (euclidean space of $n$ dimensions) each of the domains complementary to a set $M$ which is homeomorphic with an $(n-1)$-sphere, or, indeed, an $(n-1)$-manifold, is uniformly connected im kleinen. Furthermore, every point of $M$ is regularly accessible by arcs from each of the complementary domains of $M$. A generalization of the theorem that every point of an arc in $E_{3}$ is accessible from its complement (see letter from C. Kuratowski to R. L. Moore, this Bulletin, vol. 31 (1925), p. 32) is obtained as follows: Every point of an $i$-cell $(0 \leqq i \leqq n-1)$ immersed in $E_{n}$ is accessible by arcs from the complement of the cell.

\section{Professor Arnold Emch: On the geometry of symmetric} functions.

The geometric properties of symmetric substitution groups were discussed in a paper which appeared in the American Journal of Mathematics, vol. 45 (1923). In the present paper the author continues the study of symmetric functions from a geometric standpoint and investigates the properties of a number of algebraic curves which are invariant under finite collineation-groups, and also Cremona groups. The 8 cycles of order 3 on an elliptic cubic, for example, give rise to 4 elliptic class-sextics of order 12 , which bear interesting relations to the cubic. Other examples for groups $G_{12}, G_{24}, G_{48}, G_{108}$ are given.

\section{Mr. O. E. Brown: The equivalence of triples of bilinear} forms.

In the sense of Weierstrass, two triples of bilinear forms are equivalent if and only if their matrices are equivalent. We restrict the triples to those whose leading matrix is non-singular and which, thus, may be taken as the identity. Let the pairs $(I, b)$ and $(I, c)$ have the classical canonical forms $(I, \lambda)$ and $(I, \mu)$. The triple $(I, b, c)$ is equivalent to two classes of triples $\left(I, \lambda, c_{1}\right)$ and $\left(I, b_{1}, \mu\right)$ called the first and second canonical neighborhoods. A transformation reducing a matrix of the first canonical neighborhood to the second is called a transposition. The set of all transpositions is the same for all triples equivalent to a given one. 
25. Professor M. H. Ingraham: An application of $a$ theorem on matrices to the theory of equations.

This paper calls attention to the use of the fact that if $r_{i}, i=1, \cdots$, $n$, are the roots of the characteristic equation of a matrix $m$, the roots of $|g(m, x)|=0$ can be divided into sets $s_{i k}$ associated with the $r_{i}$ in such a way that $g\left(r_{i}, s_{i k}\right)=0(i, k)$, in finding an equation whose roots are connected by a polynomial equation with the roots of a given equation. All functions considered are polynomials. This is a generalization of results of A. Chatelet (Annales de l'Ecole Normale, (3), vol. 28 (1911), p. 124).

26. Professor L. E. Dickson: All integral solutions of certain diophantine equations.

Let $f(x, y)$ be a binary quadratic form whose discriminant $d$ is not a square. Given one rational solution of $f=e z^{2}$, we can express all rational solutions as the products of $\rho$ by certain quadratic functions $X, Y, Z$ of parameters $u$ and $v$, which are relatively prime integers. Express $\rho$ as an irreducible fraction $N / D$. Then our solution is integral only when $D$ divides $X, Y, Z$. It is proved that $D$ then divides $d e$. Hence all integral solutions are given by as many formulas (each involving only integral parameters) as there are positive divisors of $d e$. For certain values of $m$ and $n$ all integral solutions are obtained of the problem to find two sets of $n$ integers having the same sum, same sum of squares, $\cdots$, and same sum of $m$ th powers.

27. Professor L. E. Dickson: Binary quadratic forms with a single class in each genus.

Let the form have a negative discriminant $-\Delta$. For $\Delta$ odd, $\Delta=4 n-1$. For $a c=n, a x^{2} \pm x y+c y^{2}$ are of disciminant $-\Delta$ and are both reduced for certain $a, c$, unless $n$ is a prime or square of a prime. By using a form with the term $\pm 3 x y$, when $n$ is a prime, we conclude that $n+2$ must be a prime, a square of a prime, a triple of a prime, or 27 or 81 . By means of such results we readily obtain the few answers as far as 23,000 . The method is not quite so simple when $\Delta$ is even.

28. Professor C. C. MacDuffee: On the independence of the first and second matrices of an algebra.

This paper has appeared in full in the May-June issue of this Bulletin.

29. Professor G. A. Bliss: Expansions for implicit functions near a very general type of singularity.

In his Colloquium Lectures of 1909 the writer studied for real variables the inverse of a transformation of the form (1) $x=u+\phi_{2}+\cdots$, $y=\psi_{2}+\cdots$, where $\phi_{2}, \psi_{2}$ are homogeneous of the second degree in $u, v$ and the dots indicate terms of higher orders. The functional determinant of the second members of these equations with repect to $u, v$ will be repre- 
sented by $D(u, v)$. In a recent letter Professor J. L. Synge of the University of Dublin writes that W. R. Hamilton in his first published paper was led in 1828 to this same transformation in his effort to compare the intensity of light near a caustic surface in an optical field with the intensities in other portions of the field. By a lengthy and complicated argument, Hamilton finds that the solutions $u, v$ of equations (1) can be expressed as power series in $x,[y-\phi(x)]^{1 / 2}$, where $y=\phi(x)$ is the locus of the points in the $x y$-plane corresponding to the zeros of $D(u, v)$. In this paper the writer shows that similar ones can be deduced for singular points of implicit functions defined by the much more general equations of the form $f_{r}\left(x_{1}, \cdots, x_{n} ; y_{1}, \cdots, y_{n}\right)$ $=0,(r=1, \cdots, n)$. The singular points near which such expansions hold are of a very general type since their definition imposes only one inequality on the coefficients of the expansions of the functions $f_{r}$ besides the vanishing of the functional determinant $\left|\partial f_{r} / \partial y_{s}\right|$. Analogous results hold in the domain of real variables.

30. Professor R. E. Langer: The asymptotic location of the roots of a certain transcendental equation.

The asymptotic location of the roots of the transcendental equation of the type $\sum_{i=1}^{n} \omega_{i}(\lambda) e^{\lambda B_{i}}=0$, in which the $B_{i}$ are complex constants, has been studied by a number of authors under varying hypotheses on the coefficient functions $\omega_{i}(\lambda)$. In the most general case thus far treatedby Pólya and Schwengeler-these functions are taken to be polynomials. Wilder and Tamarkin had previously considered the case in which $\omega_{i}(\lambda)=\lambda^{r_{1}}\left\{a_{i}+E_{i}(\lambda) / \lambda\right\}$, the $a_{i}$ being constants, the $E_{i}(\lambda)$ bounded, and the $r_{i}$ being either all zero or else integers subject to special relations except when $n=2$. In this paper the equation is discussed for the general case $\omega_{i}(\lambda)=\lambda^{k_{i}}\left\{a_{i}+\epsilon_{i}(\lambda)\right\}$, where the $k_{i}$ are any real constants and $\epsilon_{i}(\lambda) \rightarrow 0$, as $|\lambda| \rightarrow \infty$. The discussion is based on the papers of Tamarkin and Wilder.

\section{Professor G. Y. Rainich: A new proof of the theorem} that the curvature field determines the electromagnetic field.

Several years ago this theorem was proved by the author, using a vector method, for all cases with exception of the singular case where the electric and magnetic vectors are equal and at right angles (Transactions of this Society, vol. 27, p. 106). In the present paper the proof is given in coordinates and the theorem is shown to be true also in the case which was left out before.

\section{Dr. H. H. Germond: Solution of a non-linear equation} of diffusion under specified boundary conditions.

The problem of the moisture distribution in an initially soaked block of wood drying from one face involves the solution of a non-linear equation of diffusion. The substitution $z=b+x /(\alpha t)^{1 / 2}$ reduces the partial differential equation to an ordinary differential equation. A solution satisfying the boundary conditions is obtained as a power series in half-powers of $z$. 
A simple approximate expression is obtained for the moisture distribution. The rate of moisture loss is deduced, and is compared with experimentally determined values.

33. Mr. N. H. McCoy: Some commutation formulas in the algebra of quantum mechanics.

For one pair of conjugate variables the special character of this algebra is due to the assumption $p q-q p=h /(2 \pi i)=c I$, where $q$ and $p$ are matrices representing the coordinate and momentum respectively and $I$ is the unit matrix. It has been shown by the author (Proceedings of the National Academy, March, 1929) that if $f$ and $\phi$ are arbitrary polynomials in $p$ and $q$,

$$
f \phi-\phi f=\sum_{s=1}^{\infty}\left(c^{s} / s !\right)\left[\partial^{s} \phi / \partial q^{s} \cdot \partial^{s} f / \partial p^{s}-\partial^{s} f / \partial q^{s} \cdot \partial^{s} \phi / \partial p^{s}\right] .
$$

This formula is extended to polynomials in $n$ pairs of variables. An application of the formula for one pair of variables to the problem of expanding functions such as $q^{m} p^{m} q^{n} p^{n \prime}$, isobaric in $p$ and $q$, in the form $\sum a_{i} p^{i} q^{i}$ gives rise to some interesting identities among binomial coefficients.

34. Mr. N. H. McCoy: On vectors whose components are quantum variables.

This paper deals with the rules of combination of vectors whose components are non-commutative quantum variables. Some analogs of the formula $p f-f p=c \partial f / \partial q$ are obtained for scalar and vector products. General commutation formulas for scalar and vector products of any two vectors are obtained by means of the results of the preceding paper.

35. Professor T. A. Pierce: Symmetric functions of $n$-ic residues $(\bmod p)$.

This paper will appear in full in an early issue of this Bulletin.

36. Dr. T. W. Moore: On the resultant of two binary forms.

This paper contains a detailed discussion of the application of the method of elimination proposed by Morley and Coble (American Journal, vol. 49 (1927), pp. 463-488) to the case of two binary forms. The forms which the resultant determinant can take in the different cases are explained and a comparison drawn with the known methods of elimination. A nonsymbolic method for writing down the resultant determinant by use of a multiple summation is furnished.

37. Professor E. G. Keller: Periodic orbits about an anchor ring.

This paper proves the existence of and constructs periodic orbits of the following types about a circular anchor ring, the radius of whose aperture and meridian sections are respectively $R-\epsilon$ and $\epsilon$. Only orbits re-entrant 
after one revolution are considered. Let $(r, \theta, z)$ and $(\rho, \theta, \phi)$ be the cylindrical and polar coordinates of the particle. Orbits exist in the meridian plane of the form $r=\rho \cos \phi, z=\rho \sin \phi$, where

$$
\rho=\sum_{i=0}^{\infty} \rho_{i}(t) \epsilon^{i}, \quad \phi=\sum_{i=0}^{\infty} \phi_{i}(t) \epsilon^{i}
$$

and where $\rho_{i}(t)$ and $\phi_{i}(t)$ are sums of sines and cosines of only even multiples of the independent variable $t$. These orbits are symmetrical with respect to both the equatorial plane and the axis of the ring. Orbits in the equatorial plane are expressible as power series in $\epsilon$ with trigonometric coefficients in $t$. Orbits inclined to the equatorial plane are of the form

$$
r=\sum_{i=0}^{\infty} r_{i}(t) \epsilon^{i}, \quad z=\sum_{i=1}^{\infty} z_{i}(t) \epsilon^{i},
$$

where $r_{i}(t)$ and $z_{i}(t)$ are either trigonometric or elliptic functions of $t$. The differential equations of motion of the particle admit one integral of areas and the vis viva integral, both of which are expressed in a finite number of terms.

38. Professor W. E. Milne: On the degree of convergence of the Gram-Charlier Series.

The author studies the series $\phi(x)=a_{0} \phi_{0}(x)+a_{1} \phi_{1}(x)+a_{2} \phi_{2}(x)+\cdots$, where $\phi_{0}(x)=(2 \pi)^{-1 / 2} e^{-x^{2} / 2}$ and where $\phi_{n}$ is the $n$th derivative of $\phi_{0}$. It is shown that if $\phi(x)$ has a continuous $k$ th derivative of bounded variation and if $x^{m} e^{x^{2 / 4}} \phi^{(k+1-m)}(x)(m=1,2, \cdots, k+1)$ are of bounded variation in the interval $(-\infty, \infty)$, then the sum of the first $n$ terms of the series will differ from $\phi(x)$ by a quantity which is $O\left(n^{-k / 2}\right)$. A similar theorem, but with lighter restrictions at infinity, is obtained for the series of orthogonal functions $f(x)=c_{0} w_{0}(x)+c_{1} w_{1}(x)+c_{2} w_{2}(x)+\cdots$ in which $w_{n}=e^{x / 4} \phi_{n}$.

39. Professor C. G. Latimer: On identities in thetafunctions.

In a former paper, the writer determined the sets of integral elements in the generalized quaternion algebras when both parameters are odd, the definition of integral element being due to Dickson. In this paper it is shown that Jacobi's fundamental identity in $\theta$-functions may be proved by employing Hurwitz' integral quaternions, and the same method is applied to the above mentioned quaternion algebras to obtain new identities in $\theta$-functions.

M. H. INGRAHAM, Associate Secretary 